\title{
Bacteriological profile, antibiotic susceptibility pattern and other factors related to neonatal meningitis: A cross-sectional hospital-based study from West Bengal
}

\author{
Kanai Lal Barik¹, Projesh Biswas², Kuntal Kanti Das ${ }^{3}$, Sumanta Laha ${ }^{4}$, Sudipto Paul ${ }^{5}$, \\ Saikat Mondal ${ }^{6}$
}

${ }^{1}$ Professor, ${ }^{2}$ Senior Resident, ${ }^{3}$ Tutor/Demonstrator, ${ }^{4}$ Associate Professor, ${ }^{5,6}$ Junior Resident, Department of Pediatric Medicine, Burdwan Medical College, East Burdwan, West Bengal, India

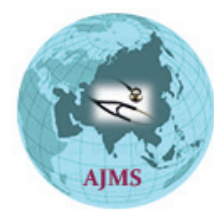

A B S T R A C T

Background: Neonatal sepsis and meningitis is an important cause of neonatal mortality and morbidity especially in the developing countries. Bacteriological profile of meningitis and antibiotic sensitivity pattern may vary from one region to another. Aims and Objectives: We have planned this study to know the etiological agent of neonatal meningitis with its antibiotic sensitivity profile and to evaluate some other associated risk factors of meningitis. Materials and Methods: This observational, cross-sectional study was done for a period of 1 year in the SNCU and NICU of a district Medical College of West Bengal in neonates presented with clinical sepsis and meningitis. Sepsis screen, blood culture, cerebrospinal fluid (CSF) study, and culture sensitivity was done and recorded along with demographic data, clinical presentation, outcome, and other associated factors. Results: We found meningitis in 55 neonates out of 250 clinical sepsis. CSF culture was positive in 42 cases with Escherichia coli (30.9\%), Klebsiella (26.1\%), Staphylococcus aureus (16.6\%), Acinetobacter (14.2\%) and Coagulase negative Staphylococcus (CoNS $11.9 \%$ ) as prevalent organism. E. coli and Klebsiella were mostly sensitive to Amikacin, Levofloxacin, and Colistin whereas less sensitive to Cefotaxime, Pipercilin-tazobactam or Meropenem and Acinetobacter showed good sensitivity only to Levofloxacin. Among the gram-positive organism, S. aureus and CoNS were only sensitive to Linezolid, Vancomycin, and Teicoplanin. Conclusion: This type of study should help to make a proper antibiotic policy for an institution so that the empirical first-line antibiotic can be started with good effect in cases of neonatal sepsis and meningitis before the arrival of culture sensitivity report.

Key words: Antimicrobial susceptibility; Etiology; Meningitis; Neonates

\section{Access this article online}

Website:

http://nepjol.info/index.php/AJMS DOI: 10.3126/ajms.v13i1.40014

E-ISSN: 2091-0576

P-ISSN: 2467-9100

Copyright (c) 2022 Asian Journal of Medical Sciences

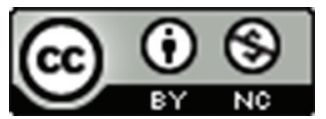

This work is licensed under a Creative Commons Attribution-NonCommercial 4.0 International License.

\section{INTRODUCTION}

Neonatal meningitis is a part of the neonatal sepsis spectrum consisting of primarily neonatal septicemia, pneumonia, meningitis, septic arthritis, or urinary tract infection. Neonatal sepsis is classified into two groups depending upon the time of onset, early-onset sepsis (EOS) which develops within $72 \mathrm{~h}$ of birth, and late-onset sepsis (LOS) after $72 \mathrm{~h}$. It is seen that etiological agents, clinical presentation, and extent of the disease varies in both groups, as in the case of neonatal meningitis, neurological features (irritability, convulsion, and bulging fontanelle) are more commonly found in those who are presented late. In a developing country like India, neonatal sepsis (30/1000 live birth) and meningitis (3/1000 live birth) are some major contributors for neonatal mortality and morbidity. ${ }^{1}$ As most cases of meningitis may not have any distinct clinical picture per se, so it is mandatory to suspect neonatal meningitis in practically all cases of suspected clinical sepsis. 
This high burden of neonatal sepsis in our country not only requires thorough investigations and management but also necessary to identify the underlying host factors and microbial factors which contribute to this so that preventive measures can be taken in time. Important host factors contributing to sepsis and meningitis are prematurity, low birth weight, prolonged rupture of the membrane, vaginal delivery without proper asepsis, etc. The microbial factors causing sepsis and meningitis of newborn is also different from the developed countries, where most of the cases of neonatal sepsis are caused by Group B Streptococcus and coagulase-negative Staphylococcus (CoNS), where as in India Klebsiella, Escherichia coli, Staphylococcus and Pseudomonas are the prevalent pathogens. ${ }^{2,3}$ As etiological agent and their antibiotic susceptibility pattern may vary in different regions of a country and also among different hospitals so knowledge of the prevalence of the local isolates and their antibiotic sensitivity pattern is essential to formulate an institution or region-based antibiotic policy. The bacteria causing neonatal sepsis also showing gradual development of resistance to various available antibiotics and it is a growing concern to all of us. The mortality of neonatal sepsis constitutes around $25 \%$ of total neonatal death in our hospital. Among the neonatal sepsis cases, death rate and disability rate are higher for neonatal meningitis. Hence, this present study is conducted to know the prevalence of local bacterial isolates from the neonates admitted with sepsis and meningitis in our sick newborn care unit (SNCU) and neonatal intensive care unit (NICU) and their antibiotic susceptibility pattern so that we can formulate a rational antibiotic policy for institution.

\section{Aims and objectives}

To know the etiological agent of neonatal meningitis with its antibiotic sensitivity profile and to evaluate some other associated risk factors of neonatal meningitis.

\section{MATERIALS AND METHODS}

This cross-sectional, observational, hospital-based study was conducted in the SNCU and NICU of a tertiary care hospital in eastern India among clinically suspected cases of neonatal sepsis admitted from March 2018 to February 2019. Of the 662 clinically suspected cases of neonatal sepsis admitted in SNCU and NICU during this time period 250 samples were taken by simple random sampling method. Neonates with major congenital anomalies, acute bilirubin encephalopathy, severe perinatal asphyxia, and suspected inborn error of metabolism were excluded from the study. After taking ethical clearance from the institution and consent from the parents, neonates are subjected to detailed history from caregivers and through clinical examination to detect sepsis and or meningitis by the clinical features such as lethargy/irritability, poor feeding/refusal to suck, temperature instability-hypo/hyperthermia, seizure, bulging fontanelle, respiratory distress/apnea, abdominal distension, poor perfusion, and periumbilical erythema. The time of onset of this presentation before or after $72 \mathrm{~h}$ helped to categorize them as early-onset or LOS. $2 \mathrm{ml}$ of venous blood was collected with IV cannula with maintaining proper asepsis before starting antimicrobial treatment, of which $1 \mathrm{ml}$ of blood was inoculated directly into Brain Heart Infusion (BHI) broth in a ratio of Blood: BHI of 1:10 in a Pediatric bact alert culture bottle. Remaining blood was utilized for sepsis screening. Around $2 \mathrm{ml}$ of cerebrospinal fluid (CSF) was collected in a sterile vial, out of which $1 \mathrm{ml}$ was inoculated in CSF culture bottle and rest of the sample was sent for biochemical, cytological, and gram staining and $\mathrm{Zn}$ staining. Blood culture bottles and CSF culture bottles were incubated for $48 \mathrm{~h}$ in BACT/ALERT machine. After $48 \mathrm{~h}$ of incubation, if BACT/ALERT machine was given a positive result then culture and sensitivity pattern was tested. Antimicrobial sensitivity test was done by modified KirbyBauer method of disc diffusion susceptibility testing, Antibiotics used for susceptibility testing are Ceftriaxone, Cefotaxime, Cefuroxime, Ceftazidime, Amikacin, Gentamicin, Linezolid, Meropenem, vancomycin, Levofloxacin, Ciprofloxacin, Imipenem, Piperacillin-Tazobactam, etc. Neonatal meningitis is defined as positive CSF culture, gram staining, or neutrophilic leucocytosis with or without low glucose $(<50 \%$ of plasma glucose level) and high protein content in a neonate with clinically suspected meningitis. Statistical analysis was done using medcalc statistical software, where we have done comparison of proportion analysis with Chi-squared test taking $\mathrm{P}<0.05$ as statistically significant.

\section{RESULTS}

Of 250 neonates, 151 (60.4\%) were term and 99 (39.6\%) were preterm. Among them, sepsis screen and blood culture were positive in $139(55.6 \%)$ and 90 cases (36\%) respectively and meningitis is present in $55(22 \%)$ neonates.

From Table 1, we can see that both clinical sepsis and neonatal meningitis are more prevalent in male $(62.8 \%$ and $60 \%)$ in comparison to female $(37.2 \%$ and $40 \%)$. However, incidence of meningitis among clinical sepsis cases is slightly more in female than male $(23.6 \%$ vs. $21 \%)$. Incidence of meningitis is more in preterm (24 out of $99,24.2 \%$ ) than term neonates (31 out of 151, 20.5\%) and slightly more among low birth weight than normal birth weight neonates $(22.7 \%$ against $21.4 \%)$. We found more cases of meningitis in the vaginal delivery group than in the cesarean section $(24.6 \%$ vs. $16.6 \%)$. Among the 250 clinically suspected neonatal sepsis cases, 110 and 140 cases were classified as 
EOS and LOS respectively. Neonatal meningitis was found in 15 cases of EOS and 40 cases of LOS thus indicative that meningitis is significantly higher among LOS than EOS group (28.5\% vs. $13.6 \%$ with $\mathrm{P}<0.05)$. Mortality is significantly higher among meningitis cases (29.1\%) in comparison to clinical sepsis $(13.2 \%)$ with $\mathrm{P}<0.05$.

From Table 2, it is seen that the positivity rate of sepsis screen, blood culture, and CSF culture are higher in the LOS meningitis group $(62.3 \%, 42.5 \%$, and $77.5 \%)$ in comparison to EOS meningitis group (53.3\%, 26.6\%, and 73.3\%). Abnormal CSF finding is also significantly higher among LOS than EOS meningitis group (84\% vs. $40 \%$ with $\mathrm{P}<0.05)$.
Table 3 shows that among the 42 CSF culture-positive case out of total 55 meningitis, most prevalent organisms are E. coli $(30.9 \%)$, Klebsiella sp. (26.1\%), and S. aureus (16.6\%) followed by Acinetobacter sp. (14.2\%) and CoNS (11.9\%). However, one significant difference is that Acinetobacter sp. $(36.3 \%)$ is the leading organism isolated from the EOS meningitis group with $\mathrm{P}<0.05$.

From Table 4, it is seen that isolated E. coli from CSF were more sensitive to Amikacin (84\%), Gentamicin (77\%), Levofloxacin (84\%), Ceftazidime (72\%), and Colistin (100\%), whereas less sensitive to Cefotaxime (38\%), Piperacillin-tazobactam (53\%), Meropenem (63\%) and netilmicin (62\%). Klebsiella spp. were sensitive to

\section{Table 1: Demographic and other factors of neonatal sepsis and meningitis}

\begin{tabular}{|c|c|c|c|c|}
\hline Variables & Clinical sepsis $(n=250)$ & Meningitis $(n=55)$ & Proportion of meningitis/clinical sepsis & P value \\
\hline \multicolumn{5}{|l|}{ Gender } \\
\hline Male & $157(62.8 \%)$ & $33(60 \%)$ & $21 \%$ & 0.595 \\
\hline Female & $93(37.2 \%)$ & $22(40 \%)$ & $23.6 \%$ & \\
\hline \multicolumn{5}{|l|}{ Gestational age } \\
\hline Preterm (<37 wks) & $99(39.6 \%)$ & $24(43.6 \%)$ & $24.2 \%$ & 0.444 \\
\hline Term ( $\geq 37$ wks) & $151(60.4 \%)$ & $31(56.4 \%)$ & $20.5 \%$ & \\
\hline \multicolumn{5}{|l|}{ Birth wt (kg) } \\
\hline$<2.5$ & $110(44 \%)$ & $25(45.5 \%)$ & $22.7 \%$ & \\
\hline \multicolumn{5}{|l|}{ Mode of delivery } \\
\hline Vaginal & $166(66.4 \%)$ & $41(74.5 \%)$ & $24.6 \%$ & 0.116 \\
\hline Caesarean & $84(33.6 \%)$ & $14(25.5 \%)$ & $16.6 \%$ & \\
\hline \multicolumn{5}{|l|}{ Onset of sepsis } \\
\hline EOS & $110(44 \%)$ & $15(27.3 \%)$ & $13.6 \%$ & 0.002 \\
\hline LOS & $140(56 \%)$ & $40(72.7 \%)$ & $28.5 \%$ & \\
\hline
\end{tabular}

Table 2: Blood and CSF findings in Early and Late onset meningitis

\begin{tabular}{|c|c|c|c|c|}
\hline Variables & $\begin{array}{l}\text { Total neonatal meningitis } \\
\qquad(n=55)\end{array}$ & $\begin{array}{l}\text { Meningitis in EOS } \\
\qquad(n=15)\end{array}$ & $\begin{array}{l}\text { Meningitis in LOS } \\
\qquad(n=40)\end{array}$ & P-value \\
\hline Sepsis screen positive & $33(60 \%)$ & $8(53.3 \%)$ & $25(62.3 \%)$ & 0.547 \\
\hline Blood culture positive & $21(38.1 \%)$ & $4(26.6 \%)$ & $17(42.5 \%)$ & 0.284 \\
\hline CSF parameters Positive & $39(70.9 \%)$ & $6(40 \%)$ & $33(84 \%)$ & 0.001 \\
\hline CSF culture positive & $42(76.3 \%)$ & $11(73.3 \%)$ & $31(77.5 \%)$ & 0.746 \\
\hline
\end{tabular}

\section{Table 3: Etiological agent of neonatal meningitis in CSF culture}

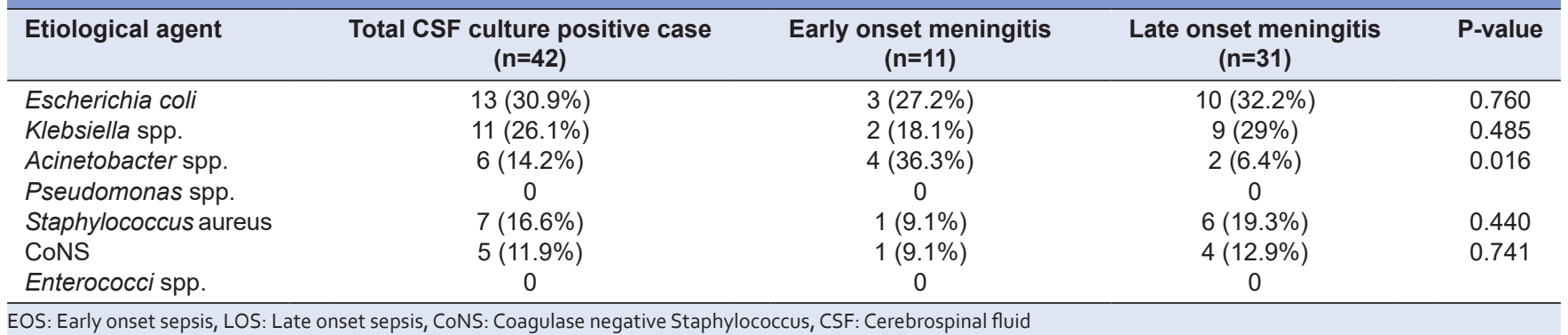




\begin{tabular}{|c|c|c|c|c|c|}
\hline Antibiotic & Escherichia coli & Klebsiella sp. & Acinetobacter Sp. & Staphylococcus aureus & CoNS \\
\hline Amikacin & $11 / 13(84 \%)$ & $7 / 11(63 \%)$ & $2 / 6(33 \%)$ & $2 / 7(28 \%)$ & $2 / 4(50 \%)$ \\
\hline Ciprofloxacin & $8 / 13(61 \%)$ & $7 / 11(63 \%)$ & $2 / 5(40 \%)$ & $1 / 6(16 \%)$ & $1 / 3(33 \%)$ \\
\hline Cefotaxime & $5 / 13(38 \%)$ & $3 / 11(27 \%)$ & $0 / 3(0 \%)$ & $0 / 5(0 \%)$ & $2 / 3(66 \%)$ \\
\hline Cefuroxime & $4 / 10(40 \%)$ & $1 / 10(10 \%)$ & $0 / 2(0 \%)$ & $0 / 3(0 \%)$ & $1 / 3(33 \%)$ \\
\hline Ceftriaxone & $9 / 13(69 \%)$ & $5 / 11(45 \%)$ & $1 / 6(16 \%)$ & $1 / 5(20 \%)$ & $3 / 5(60 \%)$ \\
\hline Ceftazidime & $8 / 11(72 \%)$ & $6 / 11(54 \%)$ & $2 / 6(33 \%)$ & $0 / 3(0 \%)$ & $0 / 1(0 \%)$ \\
\hline Cefoperazone & $5 / 9(55 \%)$ & $4 / 9(44 \%)$ & $1 / 6(16 \%)$ & $0 / 2(0 \%)$ & $1 / 3(33 \%)$ \\
\hline Colistin & $10 / 10(100 \%)$ & $7 / 8(87 \%)$ & $4 / 6(66 \%)$ & $0 / 3(0 \%)$ & $0 / 2(0 \%)$ \\
\hline Gentamicin & $10 / 13(77 \%)$ & $5 / 10(50 \%)$ & $1 / 6(16 \%)$ & $0 / 5(0 \%)$ & $1 / 4(25 \%)$ \\
\hline Imipinem-cilastatin & $2 / 5(40 \%)$ & $7 / 11(63 \%)$ & $0 / 2(0 \%)$ & $0 / 2(0 \%)$ & $1 / 5(20 \%)$ \\
\hline Levofloxacilin & $11 / 13(84 \%)$ & 9/11 (81\%) & $5 / 6(83 \%)$ & $3 / 7(42 \%)$ & $3 / 5(60 \%)$ \\
\hline Linezolid & & $0 / 2(0 \%)$ & & $5 / 7(71 \%)$ & $3 / 4(75 \%)$ \\
\hline Meropenem & $7 / 11$ (63\%) & $4 / 9(44 \%)$ & $1 / 4(25 \%)$ & $3 / 7(42 \%)$ & $2 / 3(66 \%)$ \\
\hline Netilmicin & $5 / 8(62 \%)$ & $5 / 8(62 \%)$ & $2 / 6(33 \%)$ & $0 / 4(0 \%)$ & $0 / 2(0 \%)$ \\
\hline Ofloxacin & $6 / 9(66 \%)$ & $7 / 11(63 \%)$ & $3 / 6(50 \%)$ & & \\
\hline Piperacillin-tazobactam & $7 / 13(53 \%)$ & $3 / 9(33 \%)$ & $1 / 3(33 \%)$ & $0 / 3(0 \%)$ & $0 / 2(0 \%)$ \\
\hline Vancomycin & & & & $6 / 7(85 \%)$ & $4 / 5(80 \%)$ \\
\hline Teicoplanin & & & & $3 / 3(100 \%)$ & $1 / 1(100 \%)$ \\
\hline
\end{tabular}

CoNS: Coagulase negative Staphylococcus, CSF: Cerebrospinal fluid

Levofloxacin (81\%) and Colistin (87\%) whereas most of the strain were resistant to other commonly prescribed antibiotics like Piperacillin-tazobactam (33\%), Meropenem (44\%), Cefotaxime (27\%), Amikacin (63\%), and Netilmicin $(62 \%)$. Another gram-negative organism Acinetobacter spp. Showed good sensitivity only to Levofloxacin (83\%). Among the gram-positive organisms, S. aureus and CoNS were only sensitive to Linezolid $(71 \%$ and $75 \%)$, Vancomycin (85\% and 80\%), and Teicoplanin (100\% and $100 \%)$.

\section{DISCUSSION}

Among the 250 clinically suspected neonatal sepsis cases, 157 were male and 93 were female. Hence, the male newborns outnumbered the female newborns for the development of sepsis. More susceptibility of male newborns for neonatal sepsis may be due to the presence of $\mathrm{X}$-linked immune-regulatory genes which makes $\mathrm{X}$ chromosome more immune than $\mathrm{Y}$ chromosome. ${ }^{4}$ Though out of total neonatal meningitis cases 33 were male and 22 were female, the proportion of neonatal meningitis among clinical sepsis is slightly higher in female $(23.6 \%)$ than male $(21 \%)$. Various other studies also found male newborns more susceptible for the development of sepsis and meningitis. 5,6

Among the clinical sepsis incidence of neonatal meningitis is higher in pre-term babies in our study. Hence, this is well correlated with previous studies in which preterm birth was a significant risk factor for the development of sepsis and meningitis due to their immature immune system. ${ }^{7-10}$ Similarly, we have found LBW babies with sepsis are more prone to develop neonatal meningitis than their normal birth weight counterparts like most other studies. ${ }^{9,11}$
Proportion of neonatal meningitis is much higher in LOS group than in EOS group i.e. $28.5 \%$ versus $13.6 \%$. Although Bhagat et al., found $16 \%$ of neonatal meningitis in late-onset group, ${ }^{12}$ some other references states that neonatal meningitis occurred in 5-10\% in EOS and 25\% in LOS patients. ${ }^{13}$ Hence, similar results were found in this study.

Neonatal meningitis has a very bad prognosis with case fatality rate almost $30-40 \%$ in developing countries despite improvement of neonatal intensive care. In this study, $16(29.1 \%)$ newborns died during the course of the disease and death due to gram-negative bacterial meningitis was more than the gram-positive bacterial meningitis. Different reports showed neonatal mortality rate from $15 \%$ to $26 \%$ in case of meningitis. ${ }^{14,15}$

CSF study and CSF culture was positive in $70.9 \%$ and $76.3 \%$ cases of neonatal meningitis respectively in our study. However, sepsis screen and blood culture were positive in only $60 \%$ and $38.1 \%$ cases of neonatal meningitis. So we may miss a majority of cases of neonatal meningitis unless we do CSF study and CSF culture in every case of clinically suspected neonatal sepsis. Even many cases had not shown CSF parameters changes while their CSF culture have isolated some organisms. Smith et al., found that sensitivity for predicting meningitis was low with only CSF study and emphasized the critical need for CSF culture to establish the diagnosis of meningitis. ${ }^{16}$ Visser and Hall reported normal CSF parameters in 15\% of infants with culture-proven meningitis. ${ }^{17}$ We found positivity rate of sepsis screen, blood culture, and CSF culture were more in late-onset meningitis than in early-onset meningitis in our study. 
Prevalent organism for neonatal sepsis and meningitis is different in developing countries from that of developed countries. Gram-negative bacterial sepsis is more common than the Gram-positive bacterial sepsis in developing countries like India. Bhagat et al., and Chhabra et al., found MRSA, E. coli and Klebsiella as the predominant bacteria, ${ }^{8,12}$ whereas $\mathrm{Pal}$ et al., found Klebsiella pneumonie and E. coli as most common organism causing neonatal meningitis. ${ }^{15}$ Similar result was found in the present study, where most prevalent organisms isolated in CSF culture were E. coli $(30.9 \%)$, Klebsiella sp. (26.1\%), and S. aureus $(16.6 \%)$ followed by Acinetobacter sp. and CoNS. Hence, the Gram-negative organisms outnumbered the Gram-positive organisms in causing neonatal meningitis. In a recent cohort study in Delhi, they found Acinetobacter spp, as the most common Gram-negative organism isolated which is also the most common pathogen in the EOS meningitis group in our study. ${ }^{18}$

Neonatal meningitis should be treated with appropriate antibiotics and the choice of antibiotic should depend on the sensitivity pattern of the bacteria isolated and CSF penetration of the drug. Due to the growing antibiotic resistance it is difficult to form a universal protocol but we should start the empirical antibiotics according to the local sensitivity pattern before getting the culture report. Hence, it is essential to know the sensitivity pattern of local isolates institution wise.

In the present study, most of the strains of E. coli and Klebsiella spp were resistant to common first line antibiotics such as Cefotaxime, Cefuroxime, Cefoperazone, and Piperacillin-tazobactam and few strains are also resistant to Meropenam and Imipenemcilastatin. Most of the strains were sensitive to Amikacin, Levofloxacin, and Colistin. On the other hand Gram positive organisms such as $S$. aureus and CoNS were resistant to Aminoglycosides, Fluoroquinolones, and Cephalosporin group of drugs and sensitive only to Vancomycin, Linezolid, and Teicoplanin. Studies by Sheth et al., in Western part of India and Viswanathan et al., in eastern India have also found high proportion of resistance to the common first-line antibiotics. ${ }^{19,20}$ Differences of antibiotic sensitivity and resistance patterns among various studies can be explained by the regional variation of prevalent organisms and different antibiotic regimens used for treating those organisms. ${ }^{21}$ High degree of resistance to commonly used antibiotics was also reported by Devi et al., In their study. ${ }^{7}$

\section{Limitations of this study}

This is a hospital-based study involving small number of study samples. So, the results of this study may not be applied to the others hospital or community and large scale multi-centric study is needed to establish a more generalized antibiotics sensitivity pattern.

\section{CONCLUSION}

The present study shows that low birth weight, prematurity, vaginal delivery, and LOS are associated with higher incidence of neonatal meningitis and CSF culture should be done in every cases of clinical sepsis to rule out meningitis. We have found E. coli, Klebsiella sp., S. aureus, Acinetobacter sp. and CoNS as the most prevalent organism in CSF culture, and many of them are resistant to conventional first-line antibiotics such as cephalosporin or Piperacillintazobactam. Here strains of E. coli and Klebsiella spp were mostly sensitive to Amikacin, Levofloxacin, and Colistin and Acinetobacter spp. sensitive only to Levofloxacin. Grampositive organism like $S$. aureus and CoNS were found sensitive only to Vancomycin, Linezolid, and nn. So every institution should make their own antimicrobial policy according to their local sensitivity pattern and update them at a regular interval to improve the prognosis of neonatal sepsis and meningitis by choosing appropriate antibiotics early.

\section{ACKNOWLEDGMENT}

We express our sincere thanks to everyone in the Department of Pediatric Medicine, Burdwan Medical College for their constant support and encouragement for the successful completion of the study.

\section{REFERENCES}

1. National Neonatal Perinatal Database (NNPD), Report of the National Neonatal Perinatal Database (National Neonatology Forum), 2002-2003; 2015. Available from: https://www. newbornwhocc.org/pdf/nnpd_report_2002-03.pdf [Last accessed on 2015 Mar 30].

2. Puopolo KM. Bacterial and fungal infections. Cloherty JP, Eichenwald CE and Stark RA, editors. Manual of Neonatal Care. $6^{\text {th }}$ ed. New Delhi, India: Wolters Kluwer Pvt. Ltd.; 2008. p. 275-300.

3. Singh M. Perinatal infections. In: Care of the Newborn. $6^{\text {th }}$ ed. New Delhi, India: Sagar Publication; 2004. p. 196-216.

4. Washburn TC, Medearis DN Jr. and Childs B. Sex differences in susceptibility to infections. Paediatrics. 1965;35:57-64.

5. Murthy S, Godinho MA, Guddattu V, Lewis LE and Nair NS. Risk factors of neonatal sepsis in India: A systematic review and meta-analysis. PLoS One. 2019;14(4):e0215683. https://doi.org/10.1371/journal.pone.0215683

6. Sinha A, Yokoe D and Platt R. Epidemiology of neonatal infections: Experience during and after hospitalization. Pediatr Infect Dis J. 2003;22(3):244-251. https://doi.org/10.1097/01.inf.0000055060.32226.8a

7. Devi U, Bora R, Malik V, Deori R, Gogoi B, Das JK, et al. NorthEast India. Indian J Med Res. 2017;145(1):138-143. 
https://doi.org/10.4103/ijmr.IJMR $748 \quad 15$

8. Chhabra GS, Sodhi MK and Sharma N. Clinical, hematopathological, and bacteriological profiles in neonatal septicemia and meningitis. Perinatology. 2016;17(2):55-61.

9. Kalpana L. Clinical profile of neonatal meningitis and analysis of various factors for prognostic significance. IOSR J Dent Med Sci. 2012;2(5):1-7.

https://doi.org/10.9790/0853-0250107

10. Raha BK, Baki MA, Begum T, Nahar N, Jahan N, Begum M. Clinical, bacteriological profile and outcome of neonatal sepsis in a tertiary care hospital. Med Today. 2014;26(1):18-21. https://doi.org/10.3329/medtoday.v26i1.21306

11. Furyk JS, Swann O and Molyneux E. Systemic review: Neonatal meningitis in the developing world. Trop Med Int Health. 2011;16(6):672-679.

https://doi.org/10.1111/j.1365-3156.2011.02750.x

12. Bhagat R, Hussain SQ, Gattoo IA and Wani SA. Incidence of meningitis in late onset sepsis. Int $\mathrm{J}$ Contemp Pediatr. 2015;2(2):96-102.

https://doi.org/10.5455/2349-3291.ijcp20150507

13. Moreno MT, Vargas S, Poveda R and Sáez-Llorens X. Neonatal sepsis and meningitis in a developing Latin American country. Pediatr Infect Dis J. 1994;13(6):516-520.

https://doi.org/10.1097/00006454-199406000-00010

14. Greenberg D, Shinwell ES, Yagupsky P, Greenberg S, Leibovitz E, Mazor M, et al. A prospective study of neonatal sepsis and meningitis in Southern Israel. Pediatr Infect Dis J. 1997;16(8):768-773.

https://doi.org/10.1097/00006454-199708000-00008
15. Pal AC, Sinha P, Chakraborti $S$, Mukherjee $R$ and Bandyopadhyay S. Prevalence of neonatal meningitis with special emphasis on CSF changes in cases of neonatal septicemia: A cross sectional study. IOSR J Dent Med Sci. 2016;15(9):23-27. https://doi.org/10.9790/0853-1509022327

16. Smith PB, Garges HP, Cotton CM, Walsh TJ, Clark RH and Benjamin DK Jr. Meningitis in preterm neonates: Importance of cerebrospinal fluid parameters, Am J Perinatol. 2008;25(7):421-426. https://doi.org/10.1055/s-0028-1083839

17. Visser VE and Hall RT. Lumbar puncture in the evaluation of suspected neonatal sepsis. J Pediatr. 1980;96(6):1063-1067. https://doi.org/10.1016/S0022-3476(80)80643-3

18. Investigators of the Delhi Neonatal Infection Study (Denis) Collaboration. Characterisation and antimicrobial resistance of sepsis pathogens in neonates born in tertiary care centres in Delhi, India: A cohort study. Lancet Glob Health. 2016;4(10):e752-e760. https://doi.org/10.1016/S2214-109X(16)30148-6

19. Sheth KV, Patel T and Tripathi CR. Antibiotic sensitivity pattern in neonatal intensive care unit of a tertiary care hospital of India. Asian J Pharm Clin Res. 2012;5(3):46-50.

20. Viswanathan $R$, Singh AK, Mukherjee $S$, Mukherjee R, Das $P$ and Basu S. Aetiology and antimicrobial resistance of neonatal sepsis at a tertiary care centre in eastern India: A 3 year study. Indian J Pediatr. 2011;78:409-412. https://doi.org/10.1007/s12098-010-0272-1

21. Aftab R and lqbal I. Bacteriological agents of neonatal sepsis in NICU at Nishtar Hospital Multan. J Coll Physicians Surg Pak. 2006;16(3):216-219.

\section{Authors Contribution:}

KLB - Critical revision of the manuscript; PB, KKD - Concept and design of the study, review of literature; SL - Manuscript writing, manuscript editing; SP, SM - Data acquisition, statistical analysis.

Work attributed to:

Department of Pediatric Medicine, Burdwan Medical College and Hospital, East Burdwan, West Bengal, India.

\section{Orcid ID:}

Prof. Kanai Lal Barik - (1) https://orcid.org/0000-0001-8206-7783

Dr. Projesh Biswas - (iD https://orcid.org/0000-0001-9826-372X

Dr. Kuntal Kanti Das - (1) https://orcid.org/0000-0003-3259-8222

Dr. Sumanta Laha - (i) https://orcid.org/0000-0002-8215-4737

Dr. Sudipto Paul - (i) https://orcid.org/0000-0003-4692-5845

Dr. Saikat Mondal - (1) https://orcid.org/0000-0001-5387-4201

Source of Support: None, Conflicts of Interest: None. 